\title{
Urinary Markers of Glomerular Injury in Diabetic Nephropathy
}

\author{
Abraham Cohen-Bucay ${ }^{1}$ and Gautham Viswanathan ${ }^{2}$ \\ ${ }^{1}$ Department of Medicine, St. Elizabeth's Medical Center, Tufts University School of Medicine, Boston, MA 02135, USA \\ ${ }^{2}$ Division of Nephrology, Tufts Medical Center, Boston, MA 02111, USA
}

Correspondence should be addressed to Abraham Cohen-Bucay, acbucay@gmail.com

Received 21 January 2012; Accepted 20 February 2012

Academic Editor: Omran Bakoush

Copyright ( 2012 A. Cohen-Bucay and G. Viswanathan. This is an open access article distributed under the Creative Commons Attribution License, which permits unrestricted use, distribution, and reproduction in any medium, provided the original work is properly cited.

Diabetic nephropathy, the leading cause of renal failure worldwide, affects approximately one-third of all people with diabetes. Microalbuminuria is considered the first sign and the best predictor of progression to renal failure and cardiovascular events. However, albuminuria has several limitations. Therefore, earlier, more sensitive and specific biomarkers with greater predictability are needed. The aim of this paper is to discuss the current literature on biomarkers of glomerular injury that have been implicated in diabetic kidney disease.

\section{Introduction}

Diabetes mellitus is a chronic disease that affects 366 million people worldwide $(6.4 \%$ of the adult population) and is expected to rise to 552 million by 2030 [1]. People with diabetes require at least two to three times the health-care resources compared to people who do not have diabetes, and diabetes care may account for up to $15 \%$ of national health care budgets [2]. In 2008, 1.3 million deaths were associated with diabetes [3].

Diabetes results in both microvascular and macrovascular complications. Among the microvascular complications, diabetic kidney disease is one of the most serious, with significant impact on morbidity, mortality, and quality of life [4]. Diabetic nephropathy occurs in approximately one-third of all people with diabetes and is the leading cause of renal failure in developed and developing countries [3]. Death due to renal disease is 17 times more common in diabetics than in nondiabetics [5].

Clinically, the first sign of diabetic nephropathy is considered to be microalbuminuria. As the disease progresses, patients develop macroalbuminuria, and the kidney function declines until patients end up requiring renal replacement therapy [6].

Although microalbuminuria in diabetic patients is considered to be the best predictor of progression to end-stage renal disease [7] and cardiovascular events [8-10]; earlier, more sensitive and specific markers of kidney damage might help diagnose and treat diabetic nephropathy at an earlier stage to prevent the progression to renal failure.

Diabetic nephropathy affects all the kidney cellular elements, that is, glomerular endothelia, mesangial cells, podocytes, and tubular epithelia [11]. It is characterized by excessive accumulation of extracellular matrix (ECM) with thickening of glomerular and tubular basement membranes and increased amount of mesangial matrix, which ultimately progresses to glomerulosclerosis and tubulointerstitial fibrosis [11-13]. Multiple biomarkers in serum and urine have been studied that represent different mechanisms or structural damage, based on which they have been classified as markers of glomerular injury, tubular injury, oxidative stress, inflammation, and endothelial damage $[5,12]$.

Urinary markers of glomerular damage represent either, increased permeability to plasma proteins (albumin; transferrin), or increased excretion of extracellular matrix proteins (type IV collagen; fibronectin) [13]. The former is secondary to three main factors: loss of glomerular charge selectivity [14-20], loss of glomerular size selectivity [16, 18, 21-24], or increased intraglomerular pressure [25-27]. This paper will review the literature available regarding urinary biomarkers of glomerular injury associated with diabetic nephropathy. 


\section{Albumin}

Albumin, a $65-\mathrm{kDa}$ protein produced in the liver, is the most abundant plasma protein in the body. The main functions of albumin are to regulate the oncotic pressure, to act as an acid/base buffer, and to mediate the transportation of metabolites, hormones, vitamins, and drugs [28].

In normal subjects, a small amount of albumin is filtered in the glomerulus, but almost all of it is reabsorbed by the tubules [29, 30]. Elevated urine albumin excretion (UAE) is considered a well-established marker of glomerular damage [12]. In addition, it is known that tubular dysfunction by itself may cause albuminuria owing to decreased reabsorption of filtered albumin [28].

The UAE is considered normal when it is less than $30 \mathrm{mg} /$ day or $20 \mathrm{microg} / \mathrm{min}$ (normoalbuminuria). This threshold was determined because the UAE of $95 \%$ of "normal" patients falls below this value [8]. However, it has been recognized that the risk of cardiovascular events and renal morbidity is elevated also in subjects in the "high normal" range $[8,31-33]$.

Based upon the ability of dipstick to measure urine albumin, the UAE has been classified as microalbuminuria, when the UAE is between 30 and $300 \mathrm{mg} /$ day or 20 and 200 microg/min; macroalbuminuria, when the UAE is above $300 \mathrm{mg} /$ day or $200 \mathrm{microg} / \mathrm{min}$. The rate of progression from micro to macroalbuminuria in type 2 diabetic patients is 2$3 \%$ annually [34].

Baseline albuminuria is the strongest predictor of endstage renal disease (ESRD) for type 2 diabetic patients [7]. But, not all diabetic patients develop diabetic nephropathy. Approximately 20 to $40 \%$ of diabetic patients develop microalbuminuria within $10-15$ years of diagnosis, whereas macroalbuminuria occurs within 15-20 years in 20-40\% of patients [6]. The American Diabetes Association recommends screening with annual UAE on all type 1 diabetic patients with $\geq 5$ years of disease duration and in all type 2 diabetic patients starting at diagnosis [35].

Microalbuminuria is not only a risk factor for chronic kidney disease (CKD) and ESRD, but it is also a strong predictor of total and cardiovascular mortality and cardiovascular morbidity in diabetic patients [8-10]. In patients with type 2 diabetes and nephropathy, albuminuria is the strongest risk marker for cardiovascular events [36].

Although albuminuria is widely used and is considered the best marker for renal damage in diabetic patients, certain limitations should be taken into consideration. First, not all patients with proteinuria will develop progressive renal dysfunction $[6,34]$. Additionally, $30 \%$ of diabetic patients with renal impairment have normoalbuminuria [37]. Second, the cardiovascular and renal morbidity is elevated in the "high normal" range of UAE [8, 31-33]. Third, a number of variables affecting UAE lack standardization including urine collection methods, reporting of test results, reference intervals for albumin-to-creatinine ratio and lack of a complete reference system for urine albumin and creatinine measurements [38]. Finally, multiple markers of renal dysfunction, either tubular or glomerular, can appear before the detection of microalbuminuria, suggesting that microalbuminuria occurs once significant kidney damage has already occurred $[12,39]$.

\section{Transferrin}

Transferrin is a plasma protein very similar in weight (molecular weight $76.5 \mathrm{kDa}$ ) to albumin, but slightly larger (molecular radius $4.0 \mathrm{~nm}$ compared to $3.6 \mathrm{~nm}$ of albumin). It is less anionic than albumin with an isoelectric point (pI) one unit higher, therefore, expected to be filtered more readily through the glomerular barrier. Transferrin is the major iron-binding protein in the serum, and it transports ferric ions to all proliferative cells in the body $[5,12]$.

Among type 2 diabetic patients, urinary transferrin significantly increases with respect to the progress of biopsy proven glomerular diffuse lesions [40] and has been shown that some type 2 diabetic patients with diffuse glomerular lesions without microalbuminuria had microtransferrinuria [40]. Urinary transferrin excretion has also been correlated with the degree of interstitial fibrosis, tubular atrophy, and interstitial inflammatory cell infiltration [41].

Compared to healthy controls, transferrin excretion is higher in diabetic patients, even before they develop microalbuminuria [40, 42-48]. Because diabetic patients are more likely to have transferrinuria than albuminuria $[44,49-54]$, and because the albumin/transferrin ratio was significantly smaller in normoalbuminuric and microalbuminuric compared to macroalbuminuric patients, urinary transferrin is considered to be a more sensitive marker of glomerular damage in diabetic patients [44, 50-54]. Furthermore, increased urinary transferrin excretion predicts the development of microalbuminuria in type 2 diabetic patients with normoalbuminuria $[55,56]$; in patients that already developed albuminuria, the urinary transferrin excretion has a linear relationship with UAE $[39,40,42-44,47,50,53,57-$ $60]$.

Urinary transferrin excretion is elevated in primary glomerulonephritis and other diseases that affect the glomerulus and is not specific to diabetic nephropathy $[61,62]$.

Although transferrinuria has been implicated as a cardiovascular risk factor, type 2 diabetic patients with both microalbuminuria and microtransferrinuria have a higher risk of ischemic heart disease than patients with microtransferrinuria only, suggesting that microalbuminuria may be a better predictor of ischemic heart disease than microtransferrinuria [60].

Urinary transferrin excretion is not correlated with glycemic control (hemoglobin Alc, fructosamine, and random glucose), supporting the hypothesis that transferrinuria is caused by intrinsic renal damage [42]. Nevertheless, glycemic control in newly diagnosed type 2 diabetic patients can effectively decrease transferrinuria [59]. Further evidence of transferrinuria as a marker of diabetic microvascular complications is the fact that urinary transferrin excretion is higher in type 2 diabetic patients with retinopathy [42, 44]. Conflicting results have been published regarding the correlation of urinary transferrin excretion and duration of diabetes $[42,44]$. 
Similar to albumin [63], transferrin/creatinine ratio is associated with blood pressure control [42, 44]. However, only transferrinuria, and not albuminuria, has a correlation with diurnal changes in blood pressure [64].

In type 2 diabetic patients, transferrinuria precedes tubulointerstitial changes found on biopsy [41]. It is known that reabsorption of transferrin results in release of reactive iron [57], which can produce oxidative stress on the tubular epithelium. Several studies have shown that markers of proximal tubule damage (i.e., alpha-1-microglobulin and $\mathrm{N}$ acetyl-beta-D-glucosaminidase (NAG)) and urinary transferrin excretion are associated in diabetic patients $[40,43$, $44,53,59]$. It is not clear if transferrinuria is secondary to decreased tubular reabsorption, or transferrin is the cause of tubular damage.

According to two small nonrandomized trials, low-dose angiotensin receptor blockers (ARBs) seem to prevent the progression of transferrinuria, or even reverse it, independent of their antihypertensive effect. But further randomized controlled trials are needed to support that conclusion [48, 65].

\section{Type IV Collagen}

Type IV collagen is the main constituent of both glomerular and tubular basement membranes as well as the mesangial matrix [5, 66]. Elevated glucose levels stimulate type IV collagen synthesis and may reduce its breakdown by producing advanced glycosylation of proteins. As a consequence, increased deposition of type IV collagen has been noted in the glomerular mesangial matrix of diabetic kidneys with diffuse glomerulosclerosis [5, 67, 68]. Additionally, urinary type IV collagen excretion has been associated with mesangial expansion and tubulointerstitial and glomerular injury $[69,70]$. The urinary excretion of type IV collagen correlates with the urinary excretion of other components of the glomerular basement membrane (GBM), including laminin [71]; markers of tubular damage, such as $\mathrm{N}$-acetylbeta-D-glucosaminidase (NAG) and alfa 1 microglobulin $[66,71,72]$.

Higher urinary concentrations of type IV collagen have been found in diabetics compared to controls, even in normoalbuminuric subjects [66, 70,71, 73-83], suggesting that type IV collagen could be an early predictor of diabetic nephropathy. In an Asian multicenter study of nearly 700 diabetic patients, Tomino et al. showed that the urinary excretion of type IV collagen in diabetic patients increased gradually as renal diseases progressed [82].

Multiple studies have shown that urinary excretion of type IV collagen in type 2 diabetics relates to UAE [66, 71, $72,74,78,79,82-84]$. In contrast, patients with nondiabetic chronic glomerulonephritis do not show this relationship [66].

Although type IV collagen excretion is higher in nondiabetic chronic kidney disease compared to healthy controls, type 2 diabetic patients with evidence of kidney disease have a significantly higher type IV collagen/albumin ratio compared to patients with nondiabetic nephropathy $[66,71$,
74], suggesting that urinary type IV collagen can help to differentiate diabetic versus nondiabetic nephropathy.

In a prospective study, urinary type IV collagen was found to be more sensitive than albuminuria to detect renal damage in type 2 diabetic patients [72]. However, it has been reported that as many as 33\% of microalbuminuric patients do not have increased urinary type IV collagen excretion [84]. In another study, Yagame et al. found that the area under the receiver operating characteristic (ROC) curve of albumin and type IV collagen was very similar, suggesting that UAE and urinary type IV collagen excretion have similar ability to detect early diabetic nephropathy [83].

In a follow-up study of 94 diabetic patients, Iijima et al. found that after 1 year, $25 \%$ of normoalbuminuric patients with increased urinary type IV collagen excretion developed microalbuminuria, and $75 \%$ stayed normoalbuminuric. The patients that stayed normoalbuminuric had a significant decrease in the urinary type IV collagen excretion, while the patients that developed microalbuminuria had a further increase in type IV collagen excretion [84].

Urinary type IV collagen excretion in type 2 diabetic patients is significantly associated with the duration of diabetes [71, 83]. Additionally, it is correlated with total serum cholesterol level [66] and inversely correlated with the reciprocal of serum creatinine [85]. However, it is not associated with diabetic retinopathy [71, 75]. Conflicting results have been published regarding the association of urinary type IV collagen excretion with blood pressure [71, 82, 84] and glycemic control [66, 71, 72, 74, 77, 79, 82-85].

Angiotensin-converting enzyme inhibitors (ACEIs) have been shown to decrease the type IV collagen urinary excretion in type 2 diabetic patients [86]. In contrast, low-dose ARBs failed to decrease the urinary type IV collagen excretion in a small nonrandomized, noncontrolled trial [87]. Further randomized, controlled studies are needed to conclude whether renin-angiotensin-aldosterone system blockade decreases the urinary excretion of type IV collagen.

\section{Fibronectin}

Fibronectin, a high-molecular-weight protein, is an intrinsic component of the glomerular extracellular matrix. It is produced in the liver, vascular endothelia, and platelets. Fibronectin is involved in coagulation, platelet function, and tissue repair. In diabetes it may reduce erythrocyte deformity and filterability [5].

Urinary fibronectin excretion is higher in diabetic patients compared to controls, but the difference is only significant for macroalbuminuric patients $[88,89]$. In diabetics, urinary fibronectin excretion is higher in patients with microalbuminuria compared to normoalbuminuria [88]. Additionally, urinary fibronectin levels correlate with the progression of biopsy proven glomerular diffuse lesions [90].

The excretion of urinary fibronectin degradation products correlates with UAE [91], and urinary fibronectin excretion has a weak negative correlation with creatinine clearance, mostly in patients with overt proteinuria $[88,90]$. 
Urinary fibronectin excretion might be a useful biomarker of diabetic nephropathy, but further studies are needed to determine its relevance compared to albuminuria.

\section{Laminin}

Laminin is a $900-\mathrm{kDa}$ glycoprotein that is a normal component of basement membranes. It is considered that serum laminin cannot be filtered in the normal glomerulus, and the urinary laminin is derived from the kidneys [5]. It has been shown by immunohistochemistry that laminin is located in the mesangial expansion and thickened capillary basement membranes characteristic of diabetic nephropathy [92]. As expected, urinary laminin excretion correlates with the urinary excretion of type IV collagen, the main GBM constituent [71]. Because laminin is also found in the tubular basement membrane, it could be expected to find a relationship between urinary excretion of laminin and markers of tubular injury (i.e., NAG, alfa 1 microglobulin, beta 2 microglobulin, and kappa light chains), but conflicting results have been published regarding this correlation $[71,92$, 93].

Urinary laminin excretion is higher in diabetic patients compared to healthy controls, even before the development of microalbuminuria $[71,75,93]$. However, there are conflicting results regarding the correlation of urinary laminin excretion with UAE $[71,92,93]$.

Urinary laminin excretion increases with age, specifically in patients over 60 years of age $[71,92]$. It is significantly correlated with the duration of diabetes [71], blood pressure [71], and glycemic control [71,93].

Although urinary laminin excretion is higher in nondiabetic chronic nephropathy compared to controls, type 2 diabetic patients with evidence of nephropathy had significantly higher laminin/albumin ratio compared to patients with nondiabetic nephropathy [71], suggesting that urinary laminin excretion could help differentiate diabetic versus nondiabetic nephropathy.

Further studies are needed to determine the relevance of urinary laminin excretion in diabetic nephropathy.

\section{Glycosaminoglycans}

Glycosaminoglycans (GAGs), with molecular weight ranging between 13 and $30 \mathrm{kDa}$, are important components of the extracellular matrix, cellular membranes, and endothelial glycocalyx. GAGs are involved in regulation of cell proliferation and differentiation, cell-to-matrix binding, cell-tocell interaction, and regulation of interleukin-1 production. They are also a major component of basement membranes [94]; heparan sulfate, the most prevalent glycosaminoglycan in the GBM, has been recognized as the main anionic component of the GBM $[95,96]$. In diabetes, there is a decrease in heparan sulfate content in the mesangial matrix and GBM, resulting in an alteration of the charge-selectivity of the glomerular capillaries, which may in part contribute to the proteinuria that characterizes diabetic nephropathy [5]. Hyperglycemia reduces the synthesis of GAGs by the glomerular endothelial cells decreasing the heparan sulfate content of the glycocalyx and thus increasing the passage of albumin through the glomerular capillary wall without affecting the interendothelial junctions [97]. Additionally, the systemic endothelial glycocalyx damage coincides with the development of microalbuminuria [98].

GAGs are also present in the tubular basement membrane and a correlation between urinary markers of tubular damage (beta-2 microglobulin, NAG, and Tamm-Horsfall protein) and urinary GAGs excretion has been shown [99101]. Ueta et al. report an association between urinary GAGs excretion and the severity of the GBM lesion in diabetics with good glycemic control. While in poorly controlled patients it is associated with the severity of the tubulointerstitial lesion [102].

Multiple studies have described an increased urinary GAGs excretion compared to controls, even in normoalbuminuric patients [94-96, 99, 100, 102-111]. But one study found similar urinary GAGs excretions in normoalbuminuric diabetic patients and healthy controls [102].

Discordant results have been published regarding the correlation of urinary GAGs excretion and UAE. Most of the studies found that GAGs excretion increases as albumin excretion increases $[96,99,100,108,109]$, two studies did not find a correlation $[95,106]$, and one study found a decrease in GAGs excretion as UAE increases [101]. Torffvit et al. [101] found a decrease in sulphated GAGs excretion (but not of GAGs/creatinine ratio) between normoalbuminuric and albuminuric type 1 diabetic patients. In this study they used methods to identify sulphated GAGs, while other studies have used methods that do not react with sulphated groups. Diabetes is known to induce the synthesis of hyaluronan, a nonsulphated GAGs [112]. Thus, increased urinary excretion of degraded hyaluronan can explain the increase levels of GAGs obtained in previous studies [109]. It is important to standardize the methods to measure and report urinary GAGs to conclude whether there is a correlation between urinary GAGs excretion and UAE.

Diabetic patients with manifest nephropathy have increased urinary GAGs compared to patients with incipient nephropathy $[94,99,108]$, and their sensitivity in patients with manifest nephropathy has been reported to be $100 \%$, compared to $77 \%$ of albuminuria [99].

The prevalence of diabetic macroangiopathies in diabetic patients with elevated levels of urinary GAGs is significantly higher than in those with normal levels of urinary GAGs [100]. There is also a correlation between urinary GAGs excretion an diabetic neuropathy [99]. There are conflicting results regarding the correlation of urinary GAGs excretion and diabetic retinopathy; however, even the studies that reported a positive correlation show that the urinary GAGs excretion is not an independent risk factor for diabetic retinopathy $[94,99,104,110]$.

In diabetic patients, there is a correlation between urinary GAGs excretion and blood pressure [99, 101, 106, 108], but conflicting results have been published regarding their correlation with duration of diabetes $[94,96,99,106$, $108,110]$ and glycemic control $[94,96,99,101,102,106,108$, $109]$. 
Urinary GAGs excretion could be a good marker for diabetic nephropathy and other complications of diabetes, but further studies and standardized methods of measurement of GAGs are needed before it is incorporated into clinical practice.

\section{Immunoglobulin G}

Immunoglobulin $G$ ( $\operatorname{IgG}$ ) is a protein synthesized and secreted by plasma cells than is mainly involved in the secondary immune response. It is larger than albumin, with a molecular weight of $150 \mathrm{kDa}$ and molecular radii of $62 \AA$, compared to albumin $65 \mathrm{kDa}$ and $36 \AA$, respectively [113].

Total urinary IgG excretion is higher in diabetic patients compared to controls, even before they develop microalbuminuria $[45,46,48,114-116]$. Urinary IgG excretion in normoalbuminuric diabetic patients predicts the development of microalbuminuria [56] and, unlike UAE, it correlates with the progression of glomerular diffuse lesions [117]. Intense glycemic control [115] and low-dose losartan [48] have been shown to revert the increased IgG excretion in these patients. Diurnal changes in systolic blood pressure significantly correlates with urinary IgG excretion, but not with UAE [64].

Urinary IgG excretion correlates well with urinary excretion of orosomucoid (a marker of inflammation and endothelial damage), transferrin and ceruloplasmin [114], but it has a weak and nonlinear relationship with UAE, indicating that the urinary excretion of IgG rises later and moves slower than that of albumin $[50,114]$.

Apart form using the total IgG urinary excretion as a marker of glomerular damage, the relationship between the urinary excretion of IgG and its isoform IgG4 has been used more specifically, as a marker of glomerular charge selectivity impairment. In general, the more anionic a protein is, the more difficult to pass through the glomerular barrier. Because IgG and IgG4 have similar size (strokes radius of $55 \AA$ ), but IgG4 is more anionic (isoelectric point of $\operatorname{IgG} 7.3$ and IgG4 5.8) [19], the difference in their urinary excretion would be explained only by a charge, and not size, selectivity defect. The selectivity index (SI) is the tool that has been more widely used to assess the ratio between IgG and IgG4.

In microalbuminuric patients only IgG4 excretion is elevated, exemplified by a reduced SI in microalbuminuric compared to normoalbuminuric patients $[16,118]$. While in macroalbuminuric patients excretion of both IgG and IgG4 are increased, shown by a similar IgG/IgG4 ratio but higher total IgG excretion in macroalbuminuric patients compared to microalbuminuric and normoalbuminuric patients [119, 120]. This suggests that the charge selectivity is lost in early diabetic nephropathy (microalbuminuric phase), which is difficult to evaluate in the macroalbuminuric phase because of the concomitant loss of size selectivity [14-16, 121-123].

The SI is not significantly different in normoalbuminuric diabetic patients compared to healthy controls [21, 39]; however, the urinary excretion of IgG4 and the SI has a significant correlation with UAE [17, 21, 39, 117, 124, 125]. Glycemic control [118], but not ACEI [125], increases the SI in type 1 diabetic patients with microalbuminuria. The clearance of IgG and IgG4 correlates with the duration of diabetes $[15,17]$.

The reduced SI in microalbuminuric patients does not correlate with markers of tubular injury (beta-2microglobulin) [16], but a major disadvantage of measuring $\operatorname{IgG}$ and IgG4 is that both are reabsorbed in the tubules, causing the index to reflect the tubular and glomerular handling. Additionally, local production of IgG (e.g., prostate; seminal vesicles) and low-grade urinary tract infections can be other sources of error [21].

\section{Ceruloplasmin}

Ceruloplasmin, with a molecular weight of $151 \mathrm{kDa}$, is the major copper-carrying protein in the blood. It is more negatively charged than albumin [126] and therefore more difficult to be filtered by the glomerulus.

Urinary ceruloplasmin excretion is higher in type 2 diabetic patients compared to controls [114], even in the normoalbuminuric phase $[48,115]$. It correlates well with albumin excretion rate $[126,127]$ and predicts the development of microalbuminuria in normoalbuminuric patients [56]. Glycemic control [115] and low-dose losartan [48] revert the increased urinary ceruloplasmin excretion in normoalbuminuric patients. And diurnal changes in the systolic blood pressure significantly correlate with urinary ceruloplasmin excretion, but not with UAE [64].

The ceruloplasmin/creatinine ratio is higher in diabetic nephropathy compared to nondiabetic nephropathy patients [128]. It has been reported that urine ceruloplas$\mathrm{min} /$ creatinine ratio has a sensitivity of $90-91 \%$, specificity of $61-66 \%$ and $75 \%$ concordance, in diagnosing diabetic nephropathy $[127,128]$.

Ceruloplasmin is a promising marker of diabetic nephropathy, but further studies are necessary to characterize its value compared to UAE, especially in type 1 diabetics, since all the studies have been done in type 2 diabetics.

\section{Lipocalin-Type Prostaglandin D2 Synthase}

Lipocalin-type prostaglandin D2 synthase (L-PGDS) is an enzyme-synthesizing prostaglandin D2 and a secretory protein of the lipocalin superfamily. It has similar chemical properties to albumin including anionic charge; however, it is much smaller in size (molecular weight $20-31 \mathrm{kDa}$ ), thus passing more easily through the glomerular capillary walls $[129,130]$. L-PGDS is present in the peritubular interstitium and not in the tubular cells of nondiabetic patients while, in diabetic patients, it is present in the renal tubules [131].

Urinary L-PGDS excretion is higher in patients with any form of renal disease, except for males with IgA nephropathy, compared to controls. And has a sensitivity and specificity to diagnose renal disease of 67 and 86$93 \%$, respectively [130]. It is more accurate than urinary type IV collagen, urinary markers of tubular injury (i.e., beta-2 microglobulin; NAG) and serum creatinine, but less accurate than UAE in diagnosing kidney disease [130]. 
TABLE 1: Overview of biomarkers of glomerular injury in diabetic nephropathy*.

\begin{tabular}{|c|c|c|c|c|c|c|c|}
\hline Marker & DM1 & DM2 & Prior to $\mathrm{MA}^{* *}$ & Predicts $\mathrm{MA}^{* * *}$ & UAE & $\begin{array}{c}\text { Diabetic } \\
\text { retinopathy }\end{array}$ & $\mathrm{CV}$ risk \\
\hline Transferrin & + & + & + & + & + & + & $+^{\tau}$ \\
\hline IV-C & + & + & + & & + & - & \\
\hline Fibronectin & + & + & & & + & & \\
\hline Laminin & + & + & + & & $+/-$ & & \\
\hline GAGs & + & + & + & & $+/-$ & - & + \\
\hline $\operatorname{Ig} G$ & + & + & + & + & + & & \\
\hline Ceruloplasmin & - & + & + & + & + & & \\
\hline L-PGDS & - & + & + & + & + & & + \\
\hline $\operatorname{IgM}$ & + & + & & & & & + \\
\hline
\end{tabular}

DM1: studies in type 1 diabetic patients, DM2: studies in type 2 diabetic patients, MA: microalbuminuria, UAE: correlation with urine albumin excretion, CV: cardiovascular, IV-C: type IV collagen, GAGs: glycosaminoglycans, IgG: immunoglobulin G, L-PGDS: Lipocalin-type prostaglandin D2 synthase, IgM: immunoglobulin M. *: an empty space means not enough data published, **: presence prior to microalbuminuria, ***: predicts the development of microalbuminuria, " $+/-$ ": conflicting results are published, $\tau$ : transferrin is correlated with cardiovascular risk, but albumin is a better predictor of ischemic heart disease.

Because decreased glomerular filtration rate decreases LPGDS urinary excretion, it is thought to be useful in early stages rather than advanced kidney disease [130].

Urinary L-PGDS excretion is higher in type 2 diabetic patients compared to controls, even in those without albuminuria $[131,132]$, and independently correlates with the urinary protein excretion [132]. Urinary L-PGDS excretion is useful in predicting future development of albuminuria ( $>30 \mathrm{mg} / \mathrm{gCr}$ ) in normoalbuminuric patients with a sensitivity and specificity of 56-59\% and 75-88\%, respectively [130]. Overall, L-PGDS is more accurate than urinary type IV collagen, beta 2 microglobulin, NAG, and serum creatinine in predicting proteinuria [130]. Thus, urinary L-PGDS excretion is useful to detect early renal damage in normoalbuminuric patients. Combined with albumin, it increases the power to detect diabetic nephropathy in those patients already proteinuric [130]. Glycemic control decreases LPGDS excretion to the normal range in normoalbuminuric patients [131].

In type 2 diabetic patients, the presence of higher LPGDS excretion is independently associated with history of cardiovascular disease [133].

Urinary L-PGDS has been mainly studied in type 2 diabetic patients. It is a promising novel urinary marker of kidney disease, but further studies are needed to define its role in diagnosing diabetic nephropathy.

\section{Immunoglobulin $M$}

Immunoglobulin $\mathrm{M}$ (IgM), secreted by plasma cells, is the largest antibody in the human circulatory system. Due to its large molecular radius $(120 \AA)$, the appearance of $\operatorname{IgM}$ in the urine indicates an increased density of large, highly nonselective pores ("shunts") in the glomerular capillary wall, which implicates a severe size-selectivity defect $[18,116$, 134, 135].

Increased urinary IgM excretion in patients with nondiabetic glomerular disease is associated with high degree of fibrosis and global glomerulosclerosis [134]. Furthermore, high urinary $\operatorname{IgM}$ excretion is a better predictor of decline in kidney function than albuminuria in these patients [134, 136, 137].

Increased urinary IgM excretion and IgG2/IgG4 ratio in macroalbuminuric type 2 diabetic patients compared to type 1 diabetic patients suggests that the proteinuria in type 2 diabetes is due to size-selectivity defects, while charge selectivity defects account for the proteinuria in type 1 diabetes [116].

Urinary IgM excretion is higher in macroalbuminuric type 2 diabetic patients compared to healthy controls but does not correlate with UAE or urinary alfa-1-microglobulin excretion (a marker of tubular injury) [116]. Increased urinary IgM excretion, independent of UAE, predicts cardiovascular mortality and progression to ESRD in diabetic patients $[135,138]$.

Urinary IgM excretion has not been studied as an early marker of diabetic nephropathy since it is associated with severe injury of the glomerular capillary wall. However, it is a promising marker that may predict the eventual need for renal replacement therapy and cardiovascular mortality. Urinary IgM has been mostly studied in small trials in the Nordic population and large trials in other ethnic groups are needed before it is implemented in clinical practice.

\section{Conclusion}

The current gold standard for detection and prediction of diabetic nephropathy and cardiovascular risk is albuminuria; however, it has several limitations. Makers that offer higher sensitivity and specificity for earlier detection of diabetic kidney disease and more accurate prediction of the progression to ESRD are needed. We reviewed nine biomarkers of glomerular injury implicated in diabetic kidney disease (Table 1).

Considering the results of the studies evaluating the biomarkers reviewed here, it is appealing to start utilizing them in clinical practice. However, the majority of publications reviewed are small cross-sectional studies, and there are 
only a handful of longitudinal studies. Moreover, biomarkers only have clinical value if the results are reproducible, and none of the biomarkers reviewed here have been studied in more than 2 longitudinal trials. Hence, their clinical applicability needs to be confirmed in high-quality validation studies.

Furthermore, the majority of these studies, when reporting prediction of outcomes, use odds ratios or hazard ratios, which are inaccurate to predict the risk for individual subjects. Hellemons et al. in a systematic review of longitudinal trials, have suggested the use of area under the ROC curve, positive/true-positive fractions, net reclassification improvement, integrated discrimination improvement, or the discriminative likelihood ratio as better methods to validate new markers [4].

Another methodological issue is the use of "transition in albuminuria class" as an endpoint. For example, a patient who had an increase in UAE from 25 to $35 \mathrm{mg} /$ day would be recognized as transitioning from normoalbuminuria to microalbumiruria, while a patient with increase from 35 to $295 \mathrm{mg} /$ day, would not be considered as a progressor. We have to remind ourselves that the classification of albuminuria was based on the ability of dipstick to measure urinary albumin and not the association with disease. Furthermore, it is known that risk of cardiovascular events and renal morbidity is elevated in subjects in the "high normal" range of proteinuria $[8,31-33]$. A more accurate way to report changes in albuminuria would be to assess the absolute changes in UAE.

Although many of the biomarkers reviewed here are promising, current data prevents us from making clear recommendations regarding their possible clinical use. Efforts on biomarker research should be directed at both new biomarker discovery and validation of published biomarkers on good quality, long-term, large longitudinal trials. Eventually, efforts should be made to develop a biomarker panel that is able to reliably assess diabetic nephropathy.

\section{References}

[1] International Diabetes Federation, Diabetes Atlas Fifth Edition, International Diabets Federation, Brussels, Belgium, 2011.

[2] P. Zhang, X. Zhang, J. Brown et al., "Global healthcare expenditure on diabetes for 2010 and 2030," Diabetes Research and Clinical Practice, vol. 87, no. 3, pp. 293-301, 2010.

[3] World Health Organization, Global Status Report on Noncommunicable Diseases 2010, World Health Organization, Geneva, Switzerland, 2008.

[4] M. E. Hellemons, J. Kerschbaum, S. J. Bakker et al., "Validity of biomarkers predicting onset or progression of nephropathy in patients with Type 2 diabetes: a systematic review," Diabetic Medicine, vol. 29, no. 5, pp. 567-577, 2012.

[5] C. Y. Hong and K. S. Chia, "Markers of diabetic nephropathy," Journal of Diabetes and its Complications, vol. 12, no. 1, pp. 43-60, 1998.

[6] G. Remuzzi, A. Schieppati, and P. Ruggenenti, "Nephropathy in patients with type 2 diabetes," New England Journal of Medicine, vol. 346, no. 15, pp. 1145-1151, 2002.
[7] D. de Zeeuw, D. Ramjit, Z. Zhang et al., "Renal risk and renoprotection among ethnic groups with type 2 diabetic nephropathy: a post hoc analysis of RENAAL," Kidney International, vol. 69, no. 9, pp. 1675-1682, 2006.

[8] P. Ruggenenti and G. Remuzzi, "Time to abandon microalbuminuria?” Kidney International, vol. 70, no. 7, pp. 1214-1222, 2006.

[9] S. F. Dinneen and H. C. Gerstein, "The association of microalbuminuria and mortality in non-insulin- dependent diabetes mellitus: a systematic overview of the literature," Archives of Internal Medicine, vol. 157, no. 13, pp. 1413-1418, 1997.

[10] T. Ninomiya, V. Perkovic, B. E. De Galan et al., "Albuminuria and kidney function independently predict cardiovascular and renal outcomes in diabetes," Journal of the American Society of Nephrology, vol. 20, no. 8, pp. 1813-1821, 2009.

[11] Y. S. Kanwar, J. Wada, L. Sun et al., "Diabetic nephropathy: mechanisms of renal disease progression," Experimental Biology and Medicine, vol. 233, no. 1, pp. 4-11, 2008.

[12] A. Matheson, M. D. P. Willcox, J. Flanagan, and B. J. Walsh, "Urinary biomarkers involved in type 2 diabetes: a review," Diabetes/Metabolism Research and Reviews, vol. 26, no. 3, pp. 150-171, 2010.

[13] R. Lehmann and E. D. Schleicher, "Molecular mechanism of diabetic nephropathy," Clinica Chimica Acta, vol. 297, no. 12, pp. 135-144, 2000.

[14] Y. Nakamura and B. D. Myers, "Charge selectivity of proteinuria in diabetic glomerulopathy," Diabetes, vol. 37, no. 9, pp. 1202-1211, 1988.

[15] P. Pietravalle, S. Morano, G. Cristina et al., "Charge selectivity of proteinuria in type I diabetes explored by Ig subclass clearance," Diabetes, vol. 40, no. 12, pp. 1685-1690, 1991.

[16] T. Deckert, A. Kofoed-Enevoldsen, P. Vidal, K. Norgaard, H. B. Andreasen, and B. Feldt-Rasmussen, "Size- and charge selectivity of glomerular filtration in Type 1 (insulindependent) diabetic patients with and without albuminuria," Diabetologia, vol. 36, no. 3, pp. 244-251, 1993.

[17] S. Morano, P. Pietravalle, M. G. De Rossi et al., "A charge selectivity impairment in protein permselectivity is present in type 2 diabetes," Acta Diabetologica, vol. 30, no. 3, pp. 138$142,1993$.

[18] O. Torffvit and B. Rippe, "Size and charge selectivity of the glomerular filter in patients with insulin-dependent diabetes mellitus: urinary immunoglobulins and glycosaminoglycans," Nephron, vol. 83, no. 4, pp. 301-307, 1999.

[19] A. Kofoed-Enevoldsen, W. J. Foyle, M. Fernandez, and J. S. Yudkin, "Evidence of impaired glomerular charge selectivity in nondiabetic subjects with microalbuminuria: relevance to cardiovascular disease," Arteriosclerosis, Thrombosis, and Vascular Biology, vol. 16, no. 3, pp. 450-454, 1996.

[20] M. Jeansson, A. B. Granqvist, J. S. Nyström, and B. Haraldsson, "Functional and molecular alterations of the glomerular barrier in long-term diabetes in mice," Diabetologia, vol. 49, no. 9, pp. 2200-2209, 2006.

[21] M. A. Gall, P. Rossing, A. Kofoed-Enevoldsen, F. S. Nielsen, and H. H. Parving, "Glomerular size- and charge selectivity in Type 2 (non-insulin-dependent) diabetic patients with diabetic nephropathy," Diabetologia, vol. 37, no. 2, pp. 195201, 1994.

[22] S. Friedman, H. W. Jones, and H. V. Golbetz, "Mechanisms of proteinuria in diabetic nephropathy II. A study of the size-selective glomerular filtration barrier," Diabetes, vol. 32, supplement 2, pp. 40-46, 1983. 
[23] S. Andersen, K. Blouch, J. Bialek, M. Deckert, H. H. Parving, and B. D. Myers, "Glomerular permselectivity in early stages of overt diabetic nephropathy," Kidney International, vol. 58, no. 5, pp. 2129-2137, 2000.

[24] C. Rippe, A. Rippe, O. Torffvit, and B. Rippe, "Size and charge selectivity of the glomerular filter in early experimental diabetes in rats," American Journal of Physiology, vol. 293, no. 5, pp. F1533-F1538, 2007.

[25] T. H. Hostetter, H. G. Rennke, and B. M. Brenner, "The case for intrarenal hypertension in the initiation and progression of diabetic and other glomerulopathies," American Journal of Medicine, vol. 72, no. 3, pp. 375-380, 1982.

[26] R. Zatz, T. W. Meyer, H. G. Rennke, and B. M. Brenner, "Predominance of hemodynamic rather than metabolic factors in the pathogenesis of diabetic glomerulopathy," Proceedings of the National Academy of Sciences of the United States of America, vol. 82, no. 17, pp. 5963-5967, 1985.

[27] S. Anderson and B. M. Brenner, "Pathogenesis of diabetic glomerulopathy: hemodynamic considerations," Diabetes/Metabolism Reviews, vol. 4, no. 2, pp. 163-177, 1988.

[28] H. Birn and E. I. Christensen, "Renal albumin absorption in physiology and pathology," Kidney International, vol. 69, no. 3, pp. 440-449, 2006.

[29] B. Haraldsson, J. Nyström, and W. M. Deen, "Properties of the glomerular barrier and mechanisms of proteinuria," Physiological Reviews, vol. 88, no. 2, pp. 451-487, 2008.

[30] D. Venturoli and B. Rippe, "Ficoll and dextran vs. globular proteins as probes for testing glomerular permselectivity: effects of molecular size, shape, charge, and deformability," American Journal of Physiology, vol. 288, no. 4, pp. F605F613, 2005.

[31] H. C. Gerstein, J. F. E. Mann, Q. Yi et al., "Albuminuria and risk of cardiovascular events, death, and heart failure in diabetic and nondiabetic individuals," Journal of the American Medical Association, vol. 286, no. 4, pp. 421-426, 2001.

[32] K. Wachtell, H. Ibsen, M. H. Olsen et al., "Albuminuria and cardiovascular risk in hypertensive patients with left ventricular hypertrophy: the LIFE study," Annals of Internal Medicine, vol. 139, no. 11, pp. 901-I26, 2003.

[33] K. Klausen, K. Borch-Johnsen, B. Feldt-Rasmussen et al., "Very low levels of microalbuminuria are associated with increased risk of coronary heart disease and death independently of renal function, hypertension, and diabetes," Circulation, vol. 110, no. 1, pp. 32-35, 2004.

[34] A. I. Adler, R. J. Stevens, S. E. Manley, R. W. Bilous, C. A. Cull, and R. R. Holman, "Development and progression of nephropathy in type 2 diabetes: the United Kingdom prospective diabetes study (UKPDS 64)," Kidney International, vol. 63, no. 1, pp. 225-232, 2003.

[35] American Diabetes Association, "Executive summary: standards of medical care in diabetes-2011," Diabetes Care, vol. 34, supplement 1, pp. S4-S10, 2011.

[36] D. de Zeeuw, G. Remuzzi, H. H. Parving et al., "Albuminuria, a therapeutic target for cardiovascular protection in type 2 diabetic patients with nephropathy," Circulation, vol. 110, no. 8, pp. 921-927, 2004.

[37] J. H. An, Y. M. Cho, H. G. Yu et al., "The clinical characteristics of normoalbuminuric renal insufficiency in Korean type 2 diabetic patients: a possible early stage renal complication," Journal of Korean medical science, vol. 24, pp. S75-81, 2009.

[38] W. G. Miller, D. E. Bruns, G. L. Hortin et al., "Current issues in measurement and reporting of urinary albumin excretion," Clinical Chemistry, vol. 55, no. 1, pp. 24-38, 2009.
[39] M. Yamazaki, N. Tani, K. Igarashi et al., "Changes in the glomerular pore size selectivity in patients with type II diabetes mellitus," Journal of Diabetic Complications, vol. 5, no. 2-3, pp. 138-139, 1991.

[40] M. Kanauchi, H. Nishioka, T. Hashimoto, and K. Dohi, "Diagnostic significance of urinary transferrin in diabetic nephropathy," Nippon Jinzo Gakkai shi, vol. 37, no. 11, pp. 649-654, 1995.

[41] M. Kanauchi, Y. Akai, and T. Hashimoto, "Transferrinuria in type 2 diabetic patients with early nephropathy and tubulointerstitial injury," European Journal of Internal Medicine, vol. 13, no. 3, pp. 190-193, 2002.

[42] C. K. Cheung, C. S. Cockram, V. T. F. Yeung, and R. Swaminathan, "Urinary excretion of transferrin by non-insulindependent diabetics: a marker for early complications?" Clinical Chemistry, vol. 35, no. 8, pp. 1672-1674, 1989.

[43] P. Martin, C. Walton, C. Chapman, H. J. Bodansky, and M. H. Stickland, "Increased urinary excretion of transferrin in children with Type 1 diabetes mellitus," Diabetic Medicine, vol. 7, no. 1, pp. 35-40, 1990.

[44] Y. Zhou, X. Zhang, and J. Wu, "Clinical significance of microtransferrinuria in diabetic patients," Zhonghua nei ke za zhi, vol. 36, no. 3, pp. 165-168, 1997.

[45] D. Ellis, D. J. Becker, and D. Daneman, "Proteinuria in children with insulin dependent diabetes: relationship to duration of disease, metabolic control, and retinal changes," Journal of Pediatrics, vol. 102, no. 5, pp. 673-680, 1983.

[46] O. Kordonouri, A. Jorres, C. Muller, I. Enders, G. M. Gahl, and B. Weber, "Quantitative assessment of urinary protein and enzyme excretion-a diagnostic programme for the detection of renal involvement in type I diabetes mellitus," Scandinavian Journal of Clinical and Laboratory Investigation, vol. 52, no. 8, pp. 781-790, 1992.

[47] P. Martin, H. Tindall, J. N. Harvey, T. M. Handley, C. Chapman, and J. A. Davies, "Glomerular and tubular proteinuria in Type 1 (insulin-dependent) diabetic patients with and without retinopathy," Annals of Clinical Biochemistry, vol. 29, no. 3, pp. 265-270, 1992.

[48] T. Narita, M. Hosoba, T. Miura et al., "Low dose of losartan decreased urinary excretions of IgG, transferrin, and ceruloplasmin without reducing albuminuria in normoalbuminuric type 2 diabetic patients," Hormone and Metabolic Research, vol. 40, no. 4, pp. 292-295, 2008.

[49] J. Konen, Z. Shihabi, and J. Newman, "The association of non-insulin-dependent diabetes mellitus and hypertension with urinary excretion of albumin and transferrin," American Journal of Kidney Diseases, vol. 22, no. 6, pp. 791-797, 1993.

[50] A. Bernard, A. O. Amor, J. Goemare-Vanneste et al., "Urinary proteins and red blood cell membrane negative charges in diabetes mellitus," Clinica Chimica Acta, vol. 190, no. 3, pp. 249-262, 1990.

[51] A. M. Bernard, A. A. Ouled Amor, J. Goemaere-Vanneste et al., "Microtransferrinuria is a more sensitive indicator of early glomerular damage in diabetes than microalbuminuria," Clinical Chemistry, vol. 34, no. 9, pp. 1920-1921, 1988.

[52] C. P. McCormick, J. C. Konen, and Z. K. Shihabi, "Microtransferrinuria and microalbuminuria: I. In the diabetic human," Clinical Physiology and Biochemistry, vol. 8, no. 2, pp. 53-58, 1990.

[53] M. J. O’Donnell, P. Martin, C. M. Florkowski et al., "Urinary transferrin excretion in Type 1 (insulin-dependent) diabetes mellitus," Diabetic Medicine, vol. 8, no. 7, pp. 657-661, 1991.

[54] M. J. O’Donnell, P. Martin, D. Cavan et al., "Increased urinary transferrin excretion in exercising normoalbuminuric 
insulin-dependent diabetic patients," Annals of Clinical Biochemistry, vol. 28, no. 5, pp. 456-460, 1991.

[55] T. Kazumi, T. Hozumi, Y. Ishida et al., "Increased urinary transferrin excretion predicts microalbuminuria in patients with type 2 diabetes," Diabetes Care, vol. 22, no. 7, pp. 1176 1180, 1999.

[56] T. Narita, M. Hosoba, M. Kakei, and S. Ito, "Increased urinary excretions of immunoglobulin G, ceruloplasmin, and transferrin predict development of microalbuminuria in patients with type 2 diabetes," Diabetes Care, vol. 29, no. 1, pp. 142-144, 2006.

[57] R. L. Howard, B. Buddington, and A. C. Alfrey, "Urinary albumin, transferrin and iron excretion in diabetic patients," Kidney International, vol. 40, no. 5, pp. 923-926, 1991.

[58] G. Jerums, T. J. Allen, and M. E. Cooper, "Triphasic changes in selectivity with increasing proteinuria in Type 1 and Type 2 diabetes," Diabetic Medicine, vol. 6, no. 9, pp. 772-779, 1989.

[59] M. J. O'Donnell, J. Watson, P. Martin, C. Chapman, and A. H. Barnett, "Transferrinuria in type 2 diabetes: the effect of glycaemic control," Annals of Clinical Biochemistry, vol. 28, no. 2, pp. 174-178, 1991.

[60] A. Sasaki, S. Oikawa, and T. Toyota, "Microalbuminuria is closely related to diabetic macroangiopathy," Diabetes Research and Clinical Practice, vol. 44, no. 1, pp. 35-40, 1999.

[61] M. Yaqoob, P. McClelland, A. W. Patrick, A. Stevenson, H. Mason, and G. M. Bell, "Tubulopathy with macroalbuminuria due to diabetic nephropathy and primary glomerulonephritis," Kidney International, no. 47, pp. S-101-S-104, 1994.

[62] M. Yaqoob, P. McClelland, A. W. Patrick, A. Stevenson, H. Mason, and G. M. Bell, "Tubular damage in microalbuminuric patients with primary glomerulonephritis and diabetic nephropathy," Renal Failure, vol. 17, no. 1, pp. 43-49, 1995.

[63] G. Jerums, M. E. Cooper, E. Seeman, R. M. Murray, and J. J. McNeil, "Comparison of early renal dysfunction in type I and II diabetes: differing associations with blood pressure and glycaemic control," Diabetes Research and Clinical Practice, vol. 4, no. 2, pp. 133-141, 1987.

[64] M. Hosoba, H. Fujita, T. Miura et al., "Diurnal changes in urinary excretion of IgG, transferrin, and ceruloplasmin depend on diurnal changes in systemic blood pressure in normotensive, normoalbuminuric type 2 diabetic patients," Hormone and Metabolic Research, vol. 41, no. 12, pp. 910915, 2009.

[65] S. Murayama, T. Hirano, T. Sakaue, K. Okada, R. Ikejiri, and M. Adachi, "Low-dose candesartan cilexetil prevents early kidney damage in type 2 diabetic patients with mildly elevated blood pressure," Hypertension Research, vol. 26, no. 6, pp. 453-458, 2003.

[66] S. Kado, A. Aoki, S. Wada et al., "Urinary type IV collagen as a marker for early diabetic nephropathy," Diabetes Research and Clinical Practice, vol. 31, no. 1-3, pp. 103-108, 1996.

[67] F. N. Ziyadeh, "Renal tubular basement membrane and collagen type IV in diabetes mellitus," Kidney International, vol. 43, no. 1, pp. 114-120, 1993.

[68] B. Olgemöller and E. Schleicher, "Alterations of glomerular matrix proteins in the pathogenesis of diabetic nephropathy," Clinical Investigator, vol. 71, no. 5, pp. S13-S19, 1993.

[69] H. Okonogi, M. Nishimura, Y. Utsunomiya et al., "Urinary type IV collagen excretion reflects renal morphological alterations and type IV collagen expression in patients with type 2 diabetes mellitus," Clinical Nephrology, vol. 55, no. 5, pp. 357-364, 2001.
[70] S. Ming, Z. Qi, L. Wang, and K. Zhu, "Urinary type IV collagen: a specific indicator of incipient diabetic nephropathy," Chinese Medical Journal, vol. 115, no. 3, pp. 389-394, 2002.

[71] N. Banu, H. Hara, M. Okamura, G. Egusa, and M. Yamakido, "Urinary excretion of type IV collagen and laminin in the evaluation of nephropathy in NIDDM: comparison with urinary albumin and markers of tubular dysfunction and/or damage," Diabetes Research and Clinical Practice, vol. 29, no. 1, pp. 57-67, 1995.

[72] N. Kotajima, T. Kimura, T. Kanda et al., "Type IV collagen as an early marker for diabetic nephropathy in non-insulindependent diabetes mellitus," Journal of Diabetes and its Complications, vol. 14, no. 1, pp. 13-17, 2000.

[73] N. Banu, H. Hara, S. Kataoka, G. Egusa, and M. Yamakido, "A novel method for concentrating urinary type IV collagen based on precipitation with polyethylene glycol:application to its measurement by enzyme immunoassay," Annals of Clinical Biochemistry, vol. 31, no. 5, pp. 485-491, 1994.

[74] N. Banu, H. Hara, G. Egusa, and M. Yamakido, "Serum and urinary type IV collagen concentrations in the assessment of diabetic microangiopathy," Hiroshima Journal of Medical Sciences, vol. 43, no. 4, pp. 123-133, 1994.

[75] Y. Hayashi, H. Makino, and Z. Ota, "Serum and urinary concentrations of type IV collagen and laminin as a marker of microangiopathy in diabetes," Diabetic Medicine, vol. 9, no. 4, pp. 366-370, 1992.

[76] Y. Hayashi, H. Makino, K. Shikata et al., "Increased concentrations of the basement membrane component type IV collagen in sera and urine of diabetics," Journal of Diabetic Complications, vol. 5, no. 2-3, pp. 195-196, 1991.

[77] M. Inoue, C. Oishi, Y. Shimajiri, M. Furuta, M. Ueyama, and T. Sanke, "Clinical usefulness of measurement of urine type IV collagen for detection of early phase of nephropathy in type 2 diabetic patients," Rinsho Byori, vol. 56, no. 7, pp. 564$569,2008$.

[78] R. Kikkawa, M. Togawa, M. Isono, K. Isshiki, and M. Haneda, "Mechanism of the progression of diabetic nephropathy to renal failure," Kidney International, vol. 51, no. 62, pp. S39S40, 1997.

[79] P. Sthaneshwar and S. P. Chan, "Urinary type IV collagen levels in diabetes mellitus," Malaysian Journal of Pathology, vol. 32, no. 1, pp. 43-47, 2010.

[80] T. Watanabe, K. Negishi, S. Katayama, J. Ishii, and S. Kawazu, "Serum or urinary concentration of type IV collagen in diabetics," Journal of Diabetic Complications, vol. 5, no. 2-3, pp. 191-192, 1991.

[81] N. Kotajima, Y. Fukumura, K. Obata, and I. Kobayashi, “The significance of determination of urinary type IV collagen concentrations from a random urine collection in patients with non-insulin dependent diabetes mellitus," Rinsho Byori, vol. 46, no. 3, pp. 277-282, 1998.

[82] Y. Tomino, S. Suzuki, C. Azushima et al., "Asian multicenter trials on urinary type IV collagen in patients with diabetic nephropathy," Journal of Clinical Laboratory Analysis, vol. 15, no. 4, pp. 188-192, 2001.

[83] M. Yagame, D. Suzuki, K. Jinde et al., "Significance of urinary type IV collagen in patients with diabetic nephropathy using a highly sensitive one-step sandwich enzyme immunoassay," Journal of Clinical Laboratory Analysis, vol. 11, no. 2, pp. 110116, 1997.

[84] T. Iijima, S. Suzuki, K. Sekizuka et al., "Follow-up study on urinary type IV collagen in patients with early stage diabetic 
nephropathy," Journal of Clinical Laboratory Analysis, vol. 12, no. 6, pp. 378-382, 1998.

[85] M. P. Cohen, G. T. Lautenslager, and C. W. Shearman, "Increased collagen IV excretion in diabetes: a marker of compromised filtration function," Diabetes Care, vol. 24, no. 5, pp. 914-918, 2001.

[86] M. Nishimura, T. Sasaki, A. Ohishi et al., "Angiotensinconverting enzyme inhibitors and probucol suppress the time-dependent increase in urinary Type IV collagen excretion of Type II diabetes mellitus patients with early diabetic nephropathy," Clinical Nephrology, vol. 56, no. 2, pp. 96-103, 2001.

[87] V. Woo, L. S. Ni, D. Hak et al., "Effects of losartan on urinary secretion of extracellular matrix and their modulators in type 2 diabetes mellitus patients with microalbuminuria," Clinical and Investigative Medicine, vol. 29, no. 6, pp. 365-372, 2006.

[88] M. Takahashi, "Increased urinary fibronectin excretion in type II diabetic patients with microalbuminuria," Japanese Journal of Nephrology, vol. 37, no. 6, pp. 336-342, 1995.

[89] J. A. Fagerudd, P. H. Groop, E. Honkanen, A. M. Teppo, and C. Gronhagen-Riska, "Urinary excretion of TGF- $\beta 1$, PDGF$\mathrm{BB}$ and fibronectin in insulin- dependent diabetes mellitus patients," Kidney International, vol. 51, no. 63, pp. S195S197, 1997.

[90] M. Kanauchi, H. Nishioka, T. Hashimoto, and K. Dohi, "Diagnostic significance of urinary transferrin in diabetic nephropathy," Nippon Jinzo Gakkai shi, vol. 37, no. 11, pp. 649-654, 1995.

[91] K. Kuboki, H. Tada, K. Shin, Y. Oshima, and S. Isogai, "Relationship between urinary excretion of fibronectin degradation products and proteinuria in diabetic patients, and their suppression after continuous subcutaneous heparin infusion," Diabetes Research and Clinical Practice, vol. 21, no. 1, pp. 61-66, 1993.

[92] C. Nakajima, N. Shimojo, K. I. Naka, K. Okuda, M. Yamamoto, and S. Fujii, "Clinical significance of urinary laminin P1 in diabetic patients," Journal of Diabetic Complications, vol. 5, no. 2-3, pp. 197-198, 1991.

[93] H. Miyake, K. Nagashima, H. Yagi, and K. Onigata, "Urinary laminin P1 as an index of glycemic control in children with insulin-dependent diabetes mellitus," Diabetes Research, vol. 23, no. 3, pp. 131-138, 1993.

[94] C. Hansen, A. K. Irmscher, K. Kuhlemann, J. Beyer, and G. Kahaly, "Insulin-dependent diabetes mellitus and glycosaminoglycans," Hormone and Metabolic Research, vol. 27, no. 12, pp. 555-558, 1995.

[95] J. P. H. Shield, M. Carradus, J. E. Stone, L. P. Hunt, J. D. Baum, and C. A. Pennock, "Urinary heparan sulphate proteoglycan excretion is abnormal in insulin dependent diabetes," Annals of Clinical Biochemistry, vol. 32, no. 6, pp. 557-560, 1995.

[96] D. Juretić, V. Krajnović, and J. Lukac-Bajalo, "Altered distribution of urinary glycosaminoglycans in diabetic subjects," Acta Diabetologica, vol. 39, no. 3, pp. 123-128, 2002.

[97] A. Singh, V. Friden, I. Dasgupta et al., "High glucose causes dysfunction of the human glomerular endothelial glycocalyx," American Journal of Physiology, vol. 300, no. 1, pp. F40-F48, 2011.

[98] M. Nieuwdorp, H. L. Mooij, J. Kroon et al., "Endothelial glycocalyx damage coincides with microalbuminuria in type 1 diabetes," Diabetes, vol. 55, no. 4, pp. 1127-1132, 2006.

[99] G. Kahaly, Ch. Hansen, E. Otto, G. Förster, J. Beyer, and G. Hommel, "Diabetic microangiopathy and urinary glycosaminoglycans," Experimental and Clinical Endocrinology and Diabetes, vol. 105, no. 3, pp. 145-151, 1997.

[100] I. Ueta, K. Takamatsu, K. Hashimoto et al., "[Urinary glycosaminoglycans in patients with non-insulin-dependent diabetes mellitus, collagen diseases and IgA nephropathy]," Nihon Jinzo Gakkai shi, vol. 36, no. 2, pp. 138-145, 1994.

[101] O. Torffvit, "Urinary sulphated glycosaminoglycans and Tamm-Horsfall protein in type 1 diabetic patients," Scandinavian Journal of Urology and Nephrology, vol. 33, no. 5, pp. 328-332, 1999.

[102] I. Ueta, K. Takamatsu, and K. Hashimoto, "[Urinary glycosaminoglycans in patients with incipient diabetic nephropathy]," Nihon Jinzo Gakkai shi, vol. 37, no. 1, pp. 1723, 1995.

[103] N. Bonavita, P. Reed, and P. V. Donnelly, "The urinary excretion of heparin sulfate by juvenile- and adult-onset diabetic patients," Connective Tissue Research, vol. 13, no. 1, pp. 83-87, 1984.

[104] Y. Budak, H. Demirci, M. Akdogan, and D. Yavuz, "Erythrocyte membrane anionic charge in type 2 diabetic patients with retinopathy," BMC Ophthalmology, vol. 4, p. 14, 2004.

[105] P. de Muro, P. Fresu, M. Formato et al., "Urinary glycosaminoglycan and proteoglycan excretion in normoalbuminuric patients with type 1 diabetes mellitus," Journal of Nephrology, vol. 15, no. 3, pp. 290-296, 2002.

[106] A. E. Elbert, A. M. Paglione, J. C. Bragagnolo, H. A. Mainetti, C. D. Bonavita, and M. Ruiz, "Urinary heparan sulphate is increased in normoalbuminuric diabetic patients," Medicina, vol. 60, no. 2, pp. 195-201, 2000.

[107] A. V. McAuliffe, E. J. Fisher, S. V. McLennan, D. K. Yue, and J. R. Turtle, "Urinary clycosaminoglycan excretion in NIDDM subjects: its relationship to albuminura," Diabetic Medicine, vol. 13, no. 8, pp. 758-763, 1996.

[108] A. Popławska-Kita, B. Mierzejewska-Iwanowska, M. Szelachowska et al., "Glycosaminoglycans urinary excretion as a marker of the early stages of diabetic nephropathy and the disease progression," Diabetes/Metabolism Research and Reviews, vol. 24, no. 4, pp. 310-317, 2008.

[109] G. Sindelka, J. Skrha, V. Stibor, and P. Stolba, "Glycosaminoglycans in urine of type 1 diabetic patients," Sbornik Lekarsky, vol. 94, no. 1, pp. 77-80, 1993.

[110] O. Yenice, H. Kazokoğlu, E. Özcan et al., "Erythrocyte membrane anionic content and urinary glycosaminoglycan excretion in type 1 diabetes: association with retinopathy," Current Eye Research, vol. 31, no. 11, pp. 975-981, 2006.

[111] O. Ellina, A. Chatzigeorgiou, S. Kouyanou et al., "Extracellular matrix-associated (GAGs, CTGF), angiogenic (VEGF) and inflammatory factors (MCP-1, CD 40, IFN- $\gamma$ ) in type 1 diabetes mellitus nephropathy," Clinical chemistry and laboratory medicine: CCLM / FESCC, vol. 50, no. 1, pp. 167174, 2012.

[112] P. Mahadevan, R. G. Larkins, J. R. E. Fraser, A. J. Fosang, and M. E. Dunlop, "Increased hyaluronan production in the glomeruli from diabetic rats: a link between glucoseinduced prostaglandin production and reduced sulphated proteoglycan," Diabetologia, vol. 38, no. 3, pp. 298-305, 1995.

[113] M. J. Burne, T. M. Osicka, and W. D. Comper, "Fractional clearance of high molecular weight proteins in conscious rats using a continuous infusion method," Kidney International, vol. 55, no. 1, pp. 261-270, 1999.

[114] T. Narita, H. Sasaki, M. Hosoba et al., "Parallel Increase in Urinary Excretion Rates of Immunoglobulin G, Ceruloplasmin, Transferrin, and Orosomucoid in Normoalbuminuric 
Type 2 Diabetic Patients," Diabetes Care, vol. 27, no. 5, pp. 1176-1181, 2004.

[115] T. Narita, H. Fujita, J. Koshimura et al., "Glycemic control reverses increases in urinary excretions of immunoglobulin $\mathrm{G}$ and ceruloplasmin in type 2 diabetic patients with normoalbuminuria," Hormone and Metabolic Research, vol. 33, no. 6, pp. 370-378, 2001.

[116] O. Bakoush, J. Tencer, J. Tapia, B. Rippe, and O. Torffvit, "Higher urinary IgM excretion in type 2 diabetic nephropathy compared to type 1 diabetic nephropathy," Kidney International, vol. 61, no. 1, pp. 203-208, 2002.

[117] I. Yashima, T. Hirayama, H. Shiiki, M. Kanauchi, and K. Dohi, "[Diagnostic significance of urinary immunoglobulin G in diabetic nephropathy]," Nihon Jinzo Gakkai shi, vol. 41, no. 8, pp. 787-796, 1999.

[118] H. J. Bangstad, A. Kofoed-Enevoldsen, K. Dahl-Jorgensen, and K. F. Hanssen, "Glomerular charge selectivity and the influence of improved blood glucose control in Type 1 (insulin-dependent) diabetic patients with microalbuminuria," Diabetologia, vol. 35, no. 12, pp. 1165-1169, 1992.

[119] S. Gambardella, S. Morano, A. Cancelli et al., "Urinary IgG4: an additional parameter in characterizing patients with incipient diabetic nephropathy," Diabetes Research, vol. 10, no. 4, pp. 153-157, 1989.

[120] U. di Mario, S. Morano, A. Cancelli et al., "New parameters to monitor the progression of diabetic nephropathy," American Journal of Kidney Diseases, vol. 13, no. 1, pp. 45-48, 1989.

[121] G. Cristina and G. M. De Matteis, "Early indicators of diabetic nephropathy. Changes in selective proteinuria," Recenti Progressi in Medicina, vol. 85, no. 11, pp. 540-545, 1994.

[122] T. Deckert, B. Feldt-Rasmussen, R. Djurup, and M. Deckert, "Glomerular size and charge selectivity in insulin-dependent diabetes mellitus," Kidney International, vol. 33, no. 1, pp. 100-106, 1988.

[123] S. Morano, A. Cancelli, S. Bacci et al., "The selective elimination of anionic immunoglobulins as a parameter of kidney damage in diabetes and diabetic pregnancy," Journal of Diabetic Complications, vol. 2, no. 1, pp. 2-4, 1988.

[124] Y. Chiba, N. Tani, M. Yamazaki, H. Nakamura, S. Ito, and A. Shibata, "Glomerular charge selectivity in noninsulin-dependent diabetes mellitus," Journal of Diabetic Complications, vol. 5, no. 2-3, pp. 135-137, 1991.

[125] P. M. Hansen, E. R. Mathiesen, A. Kofoed-Enevoldsen, and T. Deckert, "Possible effect of angiotensin-converting enzyme inhibition on glomerular charge selectivity," Journal of Diabetes and its Complications, vol. 9, no. 3, pp. 158-162, 1995.

[126] M. Yamazaki, S. Ito, A. Usami et al., "Urinary excretion rate of ceruloplasmin in non-insulin-dependent diabetic patients with different stages of nephropathy," European Journal of Endocrinology, vol. 132, no. 6, pp. 681-687, 1995.

[127] H. Shi, J. Fang, Y. Yang et al., "The clinical significance of detection of urinary ceruloplasmin in type 2 diabetes," Zhonghua nei ke za zhi, vol. 40, no. 12, pp. 823-825, 2001.

[128] L.-X. Qin, X. Zeng, and G. Huang, "Changes in serum and urine ceruloplasmin concentrations in type 2 diabetes," Zhong nan da xue xue bao, vol. 29, no. 2, pp. 208-211, 2004.

[129] M. Ogawa, N. Hirawa, T. Tsuchida et al., "Urinary excretions of lipocalin-type prostaglandin D2 synthase predict the development of proteinuria and renal injury in OLETF rats," Nephrology Dialysis Transplantation, vol. 21, no. 4, pp. 924934, 2006.
[130] Y. Uehara, H. Makino, K. Seiki, and Y. Urade, "Urinary excretions of lipocalin-type prostaglandin D synthase predict renal injury in type-2 diabetes: a cross-sectional and prospective multicentre study," Nephrology Dialysis Transplantation, vol. 24, no. 2, pp. 475-482, 2009.

[131] K. Hamano, Y. Totsuka, M. Ajima et al., "Blood sugar control reverses the increase in urinary excretion of prostaglandin D synthase in diabetic patients," Nephron, vol. 92, no. 1, pp. 77$85,2002$.

[132] N. Hirawa, Y. Uehara, T. Ikeda et al., "Urinary prostaglandin $\mathrm{D}$ synthase ( $\beta$-trace) excretion increases in the early stage of diabetes mellitus," Nephron, vol. 87, no. 4, pp. 321-327, 2001.

[133] R. Yoshikawa, J. Wada, K. Seiki et al., "Urinary PGDS levels are associated with vascular injury in type 2 diabetes patients," Diabetes Research and Clinical Practice, vol. 76, no. 3, pp. 358-367, 2007.

[134] O. Bakoush, O. Torffvit, B. Rippe, and J. Tencer, "High proteinuria selectivity index based upon $\operatorname{IgM}$ is a strong predictor of poor renal survival in glomerular diseases," Nephrology Dialysis Transplantation, vol. 16, no. 7, pp. 13571363, 2001.

[135] R. Tofik, O. Torffvit, B. Rippe, and O. Bakoush, "Increased urine IgM excretion predicts cardiovascular events in patients with type I diabetes nephropathy," BMC Medicine, vol. 7, p. 39, 2009.

[136] O. Bakoush, O. Torffvit, B. Rippe, and J. Tencer, "Renal function in proteinuric glomerular diseases correlates to the changes in urine IgM excretion but not to the changes in the degree of albuminuria," Clinical Nephrology, vol. 59, no. 5, pp. 345-352, 2003.

[137] O. Bakoush, M. Segelmark, O. Torffvit, S. Ohlsson, and J. Tencer, "Urine IgM excretion predicts outcome in ANCAassociated renal vasculitis," Nephrology Dialysis Transplantation, vol. 21, no. 5, pp. 1263-1269, 2006.

[138] R. Tofik, O. Torffvit, B. Rippe, and O. Bakoush, "Urine IgM-excretion as a prognostic marker for progression of type 2 diabetic nephropathy," Diabetes Research and Clinical Practice, vol. 95, no. 1, pp. 139-144, 2012. 


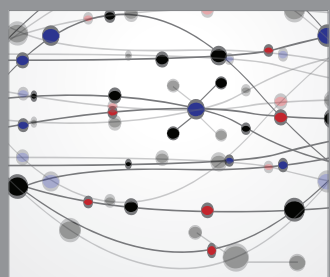

The Scientific World Journal
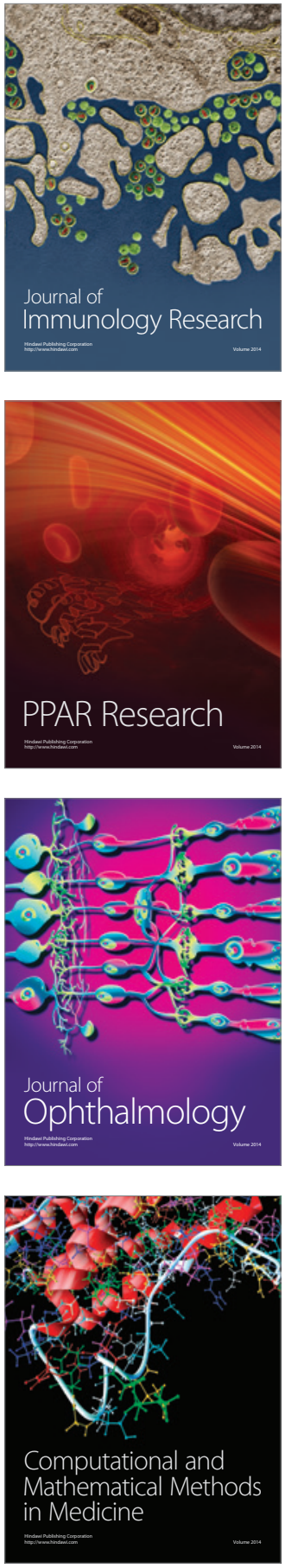

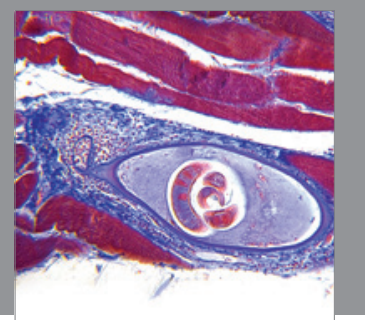

Gastroenterology

Research and Practice
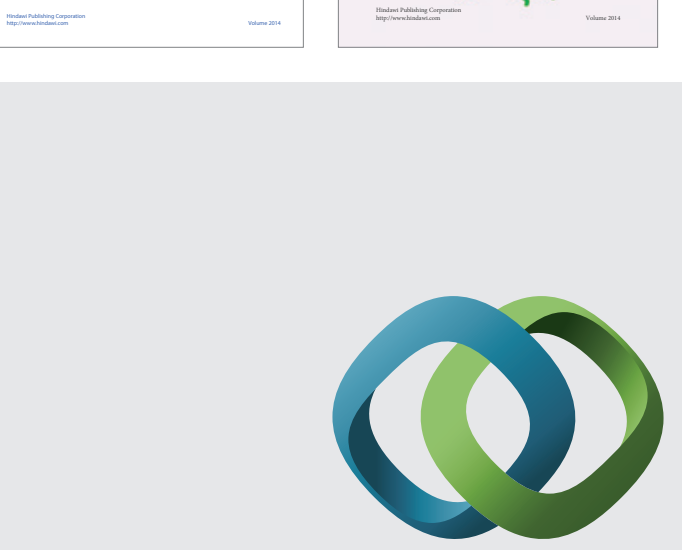

\section{Hindawi}

Submit your manuscripts at

http://www.hindawi.com
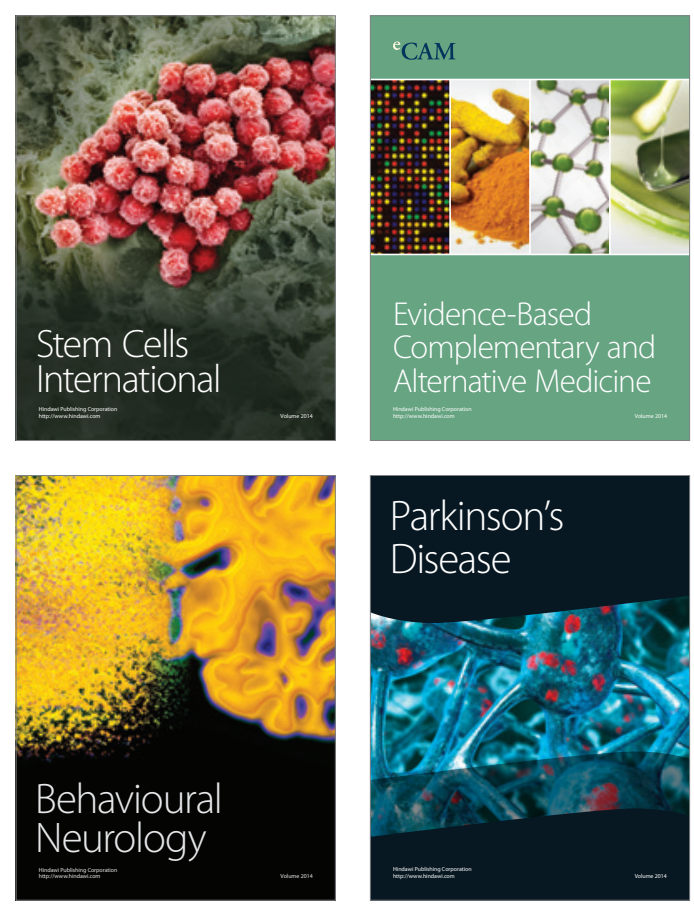

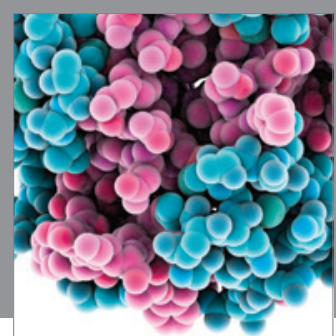

Journal of
Diabetes Research

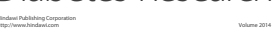

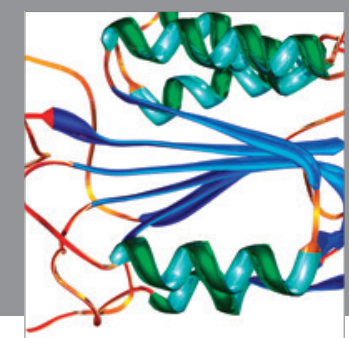

Disease Markers
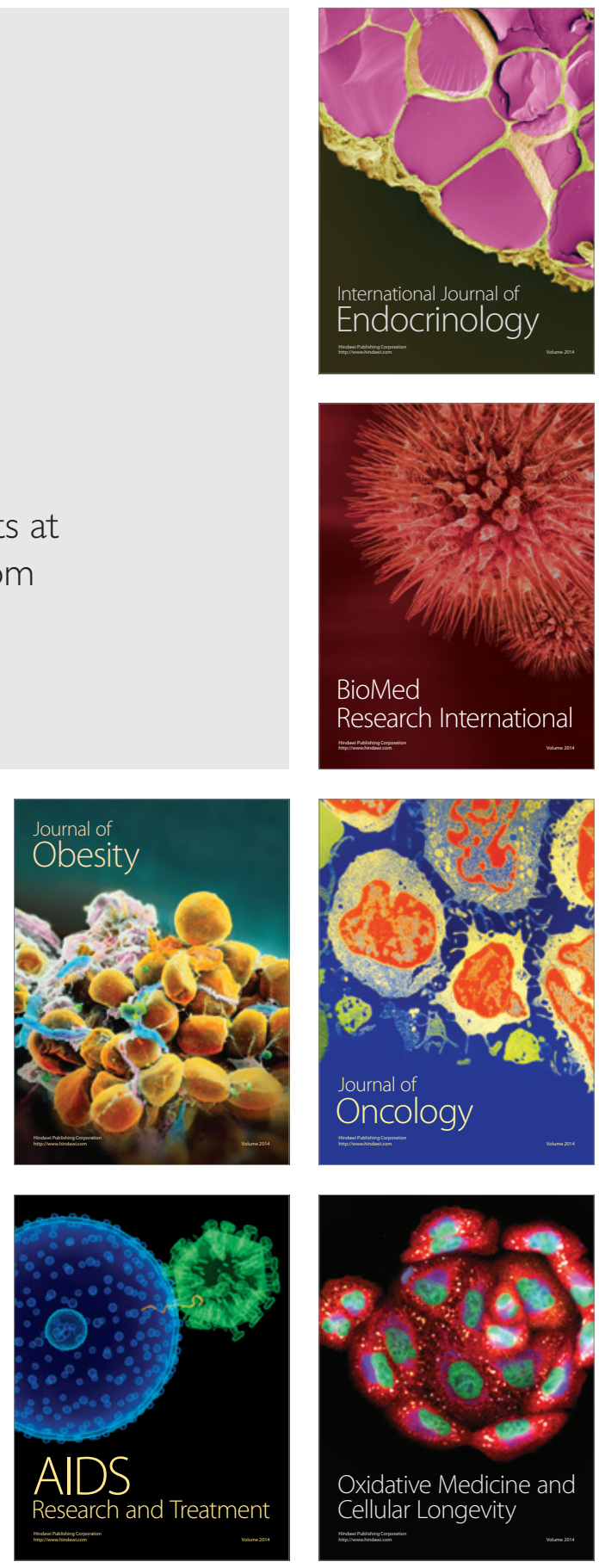